\title{
Investigating the self-healing of dynamic covalent thermoset polyimine and its nanocomposites
}

\section{Authors}

Chuanqian Shi ${ }^{1,2} \dagger$, Zhanan Zou ${ }^{2} \dagger$, Zepeng Lei ${ }^{3}$, Xingli $\mathrm{Wu}^{4}$, Zhengwei Liu $\mathrm{Lu}^{6}$, Wei Zhang ${ }^{3}$ and Jianliang Xiao ${ }^{2, *}$

\section{Affiliations}

${ }^{1}$ School of Aerospace Engineering and Applied Mechanics, Tongji University, Shanghai, 200092, China

${ }^{2}$ Department of Mechanical Engineering, University of Colorado Boulder, Colorado, 80309, USA

${ }^{3}$ Department of Chemistry, University of Colorado Boulder, Colorado, 80309, USA

${ }^{4}$ School of Mechanical Engineering, Shenyang University of Technology, Liaoning, 110870, China

${ }^{5}$ Department of Mechanical Engineering, Donghua University, Shanghai, 201600, China

${ }^{6}$ School of Mechanical-electronic and Vehicle Engineering, Weifang University, Shandong, 261061, China

$\dagger$ †. Shi and Z. Zou contributed equally to this work.

*Towhom correspondence should be addressed.Email: Jianliang.Xiao@colorado.edu 


\begin{abstract}
Self-healable and recyclable materials and electronics can improve the reliability and repairability, and can reduce environmental pollution, therefore they promise very broad applications. In this study, we investigated the self-healing performance of dynamic covalent thermoset polyimine and its nanocomposites based on dynamic covalent chemistry. Heat press was applied to two laminating films of polyimine and its nanocomposites to induce self-healing. The effects of heat press time, temperature and load on the interfacial shear strength of re-healed films were investigated. The results showed that increasing the heat press time, temperature and load can significantly improve the interfacial shear strength and thus the self-healing effect. For polyimine nanocomposites, increasing the heat press time, temperature and load led to improved electrical conductivity of the re-healed films.
\end{abstract}




\section{Introduction}

Realizing self-healing in materials, devices and structures can offer a lot of benefits on reliability, durability, cost, functionality, and performance [1-3]. To achieve self-healability, various strategies have been investigated, including emitting microvascular agents[4], hydrogen bonds[5], and dynamic covalent bonds[6-9]. Depending on the required stimulation and the nature of the self-healing process, selfhealing materials can be divided into autonomic and non-autonomic self-healing materials[3,10,11]. Autonomic self-healing materials automatically respond to damage even without force or heat applied [12]. In contrast, non-autonomic self-healing materials need modest external stimulation, such as heat[13-17], chemicals[9,18-20], light[21-24], pressure[18,25], water[26-28] and magnetic field[29,30]. To introduce conductivity into the self-healable polymers for functional devices, an effective method is to physically mix conductive materials into the network, such as silver nanoparticles and nanowires, and carbon nanotubes [31-33]. Various applications of such strategy can be found in flexible electronics, optical devices, artificial skins[9,12,33-36], antennas[37], stretchable wires[25], and artificial muscles[38], and flexible electronics[39-43].

Among the many self-healing mechanisms, dynamic covalent bonding in polymer networks usually offers higher robustness, mechanical strength and stability. Heat press, in this case, is a widely-adopted method for self-healing of polymers[4439]. Researchers demonstrated a series of dynamic covalent thermoset polyimine and composites that can self-heal under heat press [7,4045,4146]. More recently, we 
reported a self-healable, recyclable and malleable electronic skin, based on the dynamic covalent bonding polyimine and its silver nanoparticle (AgNP) nanocomposites [9]. However, the dependence of the self-healing property of polyimine and its nanocomposites on the heat press condition was not investigated. In this paper, we present results on how the heat press temperature, load, and time affect the self-healing of polyimine and its nanocomposites. The effect of weight ratio of AgNPs in the nanocomposites is also discussed. The interfacial shear strength of the re-healed polyimine and its nanocomposites, as well as the electrical conductivity of the re-healed polyimine nanocomposites, under different heat press conditions were characterized and presented.

\section{Synthesis and self-healing experiment}

2.1 Synthesis and self-healing process. Pure polyimine films were prepared from commercially available monomers, as schematically illustrated in Fig. 1a. A mixture of diethylenetriamine $(0.092 \mathrm{~g}, 0.895 \mathrm{mmol})$ and tris(2-aminoethyl)amine (0.204g, $1.39 \mathrm{mmol})$ was dissolved in ethanol $(5 \mathrm{~mL})$ and added to a $20 \mathrm{~mL}$ vial. For polyimine nanocomposites, silver nanoparticles in different weight ratios ( $0 \% \mathrm{wt} 0 \mathrm{~g}$, 20\%wt 0.348g, 50\%wt 0.696g) were added into the vial and sonicated for 3 hours at this stage. Then, terephthaldehyde $(0.4 \mathrm{~g}, 2.98 \mathrm{mmol})$ was added into the vial, and the mixed solution was poured into a petri dish coated with PDMS. The solvent was then allowed to evaporate for 12 hours in a fume hood under ambient conditions. The detailed self-healing process of a pure (composite) polyimine film is schematically 
illustrated in Fig. 1b, with optical images of the pure polyimine film shown at the bottom of each frame. A virgin polyimine strip $(0.15 \mathrm{~mm} \times 3.5 \mathrm{~mm} \times 36 \mathrm{~mm}$, Fig. $1 \mathrm{~b}$, top left) was cut broken along its width direction (Fig. 1b, top right). By overlapping $2 \mathrm{~mm}$ at the cut area and applying heat press (Fig. 1b, bottom right), new covalent bonds were formed between the two polyimine films, driven by the dynamic bonding interactions in polyimine networks, leading to re-healed interface $(0.15 \mathrm{~mm} \times 3.5 \mathrm{~mm} \times 34 \mathrm{~mm}$, Fig. $1 \mathrm{~b}$, bottom left).

2.2 Interface of self-healed polyimine and nanocomposite films. Optical microscopy images of the cross sections of the overlapping areas of re-healed polyimine and nanocomposites in Fig. 2 show the interfaces at different stages of the self-healing process. Figures 2a, 2b and 2c show the images of the pure polyimine, polyimine nanocomposites with 20\%wt AgNPs and with 50\%wt AgNPs, respectively. The left frame in each figure exhibits the interface of overlapped films before heat press. A gap of width $\sim 20 \mu \mathrm{m}$ can be clearly seen. After heat press at $80^{\circ} \mathrm{C}$ with $500 \mathrm{~g}$ load for 5 minutes, a discernible trace of interface can be observed in the middle frames of Figs. 2a, $2 \mathrm{~b}$ and 2c, but the gap has disappeared. Because of the covalent bonding formation at the interface, the self-healed polyimine and nanocomposite films can hold 1g weights, as shown in the insets of the middle frames of Figs. 2a, 2b and 2c. Increasing the time of heat press allows for more covalent bonds to be formed across the interface, leading to better self-healing effect. As shown in the right frames of Figs. 2a, 2b and 2c, after 65 minutes of heat press at $80^{\circ} \mathrm{C}$ with $500 \mathrm{~g}$ load, the interfaces are indiscernible. 


\section{Interfacial strength of self-healed polyimine and nanocomposite films}

\subsection{Uniaxial tension test of self-healed polyimine and nanocomposite films.}

To quantitatively characterize the self-healing effect, uniaxial tension tests were conducted to compare the shear strength of re-healed pure and conductive polyimine nanocomposite films with different heat press conditions. Shear failure of the lap-joint area should be the primary failure mode in the uniaxial tension test, and the shear strength can be obtained by recording the failing load per unit shear area[4z그]. As illustrated in Fig. 3a, long $(\sim 0.3 \mathrm{~mm} \times 3.5 \mathrm{~mm} \times 17 \mathrm{~mm})$ and short $(\sim 0.45 \mathrm{~mm} \times 3.5 \mathrm{~mm} \times 3 \mathrm{~mm})$ aluminum alloy plates were bonded to the self-healed polyimine film strips. The long aluminum alloy plates could increase tensile strength and avoid the failure of test samples outside of the lap-joint areas. -The short aluminum alloy plates can adjust the loading axis to be aligned with the center lines of the specimens. Figures 3b-3d display optical microscopy images of the interface areas of failed pure (Fig. 3b) and nanocomposite polyimine films (Fig. 3c, 20\%wt AgNPs, and Fig. 3d, 50\%wt AgNPs), which were re-healed at $80^{\circ} \mathrm{C}$ with $500 \mathrm{~g}$ load for 5 minutes before the tension test. Optical images of the failed film specimens are shown at the bottom of each frame. From the optical microscope images of the failed film specimens in Figures 3b-3d, the proportion of the damage areas to the overlapping interfaces was $2.9 \% \pm 3 \%$, no visible damages can be seen in the failed interface areas, indicating partial self-healing. Figures 3e-3g present optical microscopy images of the interface areas of failed pure (Fig. 3e) and nanocomposite polyimine films (Fig. 3f, 
20\%wt AgNPs, and Fig. 3g, 50\%wt AgNPs), which were self-healed at $80^{\circ} \mathrm{C}$ with $500 \mathrm{~g}$ load for 65 minutes before the tension test. Optical images of the failed film specimens are shown at the bottom of each frame. Material damages in the interface areas can be clearly seen, the damage area ratio was $46.5 \% \pm 14 \%$ as demonstrated in the optical microscopy images. This indicates that sufficient covalent bonds were formed at the interfaces during heat press, leading to interfaces with mechanical strength comparable to the virgin material.

\subsection{Dependency of interfacial shear strength of polyimine and}

nanocomposite films on heat press conditions. The results of uniaxial tension test are presented in Fig. 4, which compares the interfacial shear strength of self-healed pure and nanocomposite polyimine films with different heat press conditions. The effect of heat press time is exhibited in Fig. 4a, with constant heat press temperature at $80{ }^{0} \mathrm{C}$ and load at $500 \mathrm{~g}$. For pure polyimine, the interfacial shear strength of self-healed films increases monotonically with heat press time, from $1.8 \mathrm{MPa}$ after 5 minutes heat press to 9.8 $\mathrm{MPa}$ after 65 minutes heat press. Same trend is observed for polyimine nanocomposite films, however, higher AgNP weight ratio leads to lower shear strength of self-healed films when heat pressed under the same condition for the same time. For example, the shear strength of the self-healed polyimine nanocomposite films with 50\%wt AgNP increases from 1.4 MPa after 5 minutes heat press to 8.8 MPa after 65 minutes heat press, which are reduced by $23 \%$ and $11 \%$ compared with pure polyimine films which are $23 \%$ and $11 \%$ compared with pure polyimine films. This indicates that 
adding AgNPs to the polyimine network introduces conductivity at the expense of decreasing self-healing effectiveness. In addition to time, the effects of heat press temperature and load on the interfacial shear strength of self-healed pure and nanocomposite polyimine films were also investigated. Figure $4 \mathrm{~b}$ exhibits the increase of interfacial shear strength of self-healed films with heat press temperature, while the heat press load and time were kept constant at $500 \mathrm{~g}$ and 25 minutes. Still, the selfhealed nanocomposite films have lower shear strength compared with the pure polyimine films, when they are self-healed under the same heat press conditions. For example, the shear strength of self-healed nanocomposite films with 50\%wt AgNPs are 3.0 MPa and 8.6 MPa under $60^{\circ} \mathrm{C}$ and $115^{\circ} \mathrm{C}$ heat press, which are $43 \%$ and $35 \%$ lower than those of self-healed pure polyimine films with the same heat press conditions. Figure 4c presents results of the effect of heat press load on the shear strength of selfhealed films. It shows that the shear strength increases with increasing heat press load, while the heat press temperature and time were kept constant at $80{ }^{\circ} \mathrm{C}$ and 25 minutes. When the heat press load increases from $50 \mathrm{~g}$ to $1100 \mathrm{~g}$, the shear strength of self-healed pure polyimine films increases from 1.9 MPa to 9.4 MPa, and that of self-healed nanocomposite films with 20\%wt AgNPs increases from 0.6 MPa to 8.6 MPa. For nanocomposite films with 50\%wt AgNPs, heat press with 50 g load didn’t yield effective self-healing at the interface. For $200 \mathrm{~g}$ and $1100 \mathrm{~g}$ heat press loads, the interfacial shear strengths of self-healed films are 3.8 MP and 7.5 MPa, 39\% and 20\% lower than those of self-healed pure polyimine films. 
For the polyimine studied here, the mechanism for self-healing on the molecular level is the dynamic covalent bond exchange reactions under environmental stimulation. With increasing heat press temperature, the bond exchange reactions are happening at a faster rate, leading to more effective interfacial healing given the same load and time. For the same heat press temperature and load, more time allows for more covalent bonds to be formed at the interface, leading to stronger bonding. The heat press load ensures effective contact at the interface. Increasing the heat press load increases effective contact area, which allows for more covalent bonds to be formed at the interface given the same heat press temperature and time [4348-4550]. For the polyimine nanocomposite, the existence of nanoparticles impedes the bond exchange reactions at the interface, leading to less effective interfacial bonding. That's why the shear strength of the self-healed films decreases with the increasing AgNP weight ratio.

\section{Conductivity of self-healed nanocomposite films}

The characteristics of the conductivity of self-healing polyimine nanocomposite films are presented in Fig. 5. When a strip of conductive polyimine nanocomposite film was integrated into a simple lighting circuit, as illustrated in Fig. 5a, the LED light turned on (Fig. 5b, top left). When the polyimine film was cut in the middle, it caused the circuit to disconnect and the LED to turn off (Fig. 5b, top right). After heat pressing the polyimine films at $500 \mathrm{~g}$ load and $80{ }^{\circ} \mathrm{C}$ for 5 mins, the conductive polyimine film was healed and the LED light turned on again (Fig. 5b bottom). The dependency of the conductivity of the self-healed polyimine nanocomposite films on the heat press 
condition was also studied, and the results are presented in Figs. 5c-5e. The electrical resistances of the self-healed (denoted by $\mathrm{R}$ ) and virgin (denoted by $\mathrm{R}_{0}$ ) polyimine nanocomposite films were measured using four-point method. Figure 5c exhibits the effect of the heat press time on the electrical resistances of self-healed polyimine films, which are normalized by those of the virgin films with 20\%wt and 50\%wt AgNPs. Here, the heat press temperature and load were kept constant at $80{ }^{\circ} \mathrm{C}$ and $500 \mathrm{~g}$, respectively. Clearly, the electrical resistance decreases with increasing heat press time, and higher AgNP weight ratio leads to lower electrical resistance. The effects of heat press temperature and load on the electrical resistance of self-healed films are presented in Figs. 5d and 5e, respectively, while the other heat press conditions were kept constant. The results show consistent phenomena as the shear strength measurements. When the other heat press conditions were kept constant, increasing the heat press temperature and load led to more effective bond exchange reactions and better bonding at the interface. This caused the decrease of the electrical resistance at the interface.

\section{Conclusion}

In conclusion, this study revealed that the heat press condition and AgNP weight ratio can affect the self-healing of dynamic covalent thermoset polyimine and its nanocomposites. The effect of heat press time, temperature, and load, and AgNP weight ratio on self-healing of polyimine and its nanocomposites were experimentally investigated. The results have shown that increasing the heat press time, temperature and load leads to more effective bond exchange reactions and better bonding at the 
interface, which increases the interfacial shear strength of the self-healed polyimine and its nanocomposites. Introducing AgNPs into the polyimine network provides electrical conductivity at the expense of lower self-healing performance, as the AgNPs impedes bond exchange reactions at the interface. Increase of AgNP weight ratio leads to decrease of electrical resistance and shear strength of the self-healed polyimine nanocomposite films.

\section{Acknowledgments:}

Financial support from NSF (Grant No. CMMI-1762324) is gratefully acknowledged.

\section{References:}

[1] Blaiszik, B. J., Kramer, S. L. B., Olugebefola, S. C., Moore, J. S., Sottos, N. R., and White, S. R., 2010, "Self-Healing Polymers and Composites,” Annu. Rev. Mater. Res., 40, pp. 179-211.

[2] Yuan, Y. C., Yin, T., Rong, M. Z., and Zhang, M. Q., 2008, "Self Healing in Polymers and Polymer Composites. Concepts, Realization and Outlook: A Review,” Express Polym. Lett., 2(4), pp. 238-250.

[3] Thakur, V. K., and Kessler, M. R., 2015, “Self-Healing Polymer Nanocomposite Materials: A Review,” Polymer., 69, pp. 369-383.

[4] Toohey, K. S., Sottos, N. R., Lewis, J. A., Moore, J. S., and White, S. R., 2007, “Self-Healing Materials with Microvascular Networks,” Nat. Mater., 6(8), pp. 581-585.

[5] Su, C., Acik, M., Takai, K., Lu, J., Hao, S. J., Zheng, Y., Wu, P., Bao, Q., Enoki, T., Chabal, Y. J., and Loh, K. P., 2012, "Probing the Catalytic Activity of Porous Graphene Oxide and the Origin of This Behaviour," Nat. Commun., 3, p. 1298.

[6] Bollinger, J., Britton, J., Gisin, N., Knight, P., Kwiat, P., and Percival, I., 2001, “Autonomic Healing of Polymer Composites,” Nature, 409(6822), pp. 794797.

[7] Taynton, P., Yu, K., Shoemaker, R. K., Jin, Y., Qi, H. J., and Zhang, W., 2014, 
“Heat- or Water-Driven Malleability in a Highly Recyclable Covalent Network Polymer,” Adv. Mater., 26(23), pp. 3938-3942.

[8] Li, Y., Chen, S., Wu, M., and Sun, J., 2012, "Polyelectrolyte Multilayers Impart Healability to Highly Electrically Conductive Films,” Adv. Mater., 24(33), pp. 4578-4582.

[9] Zou, Z., Zhu, C., Li, Y., Lei, X., Zhang, W., and Xiao, J., 2018, “Rehealable, Fully Recyclable, and Malleable Electronic Skin Enabled by Dynamic Covalent Thermoset Nanocomposite,” Sci. Adv., 4(2), p. eaaq0508.

[10] Williams, K. A., Dreyer, D. R., and Bielawski, C. W., 2008, “The Underlying Chemistry of Self-Healing Materials,” Mrs Bull., 33(8), pp. 759-765.

[11] Hager, B. M. D., Greil, P., Leyens, C., Zwaag, S. Van Der, and Schubert, U. S., 2010, “Self-Healing Materials,” Adv. Mater., 22(47), pp. 5424-5430.

[12] Son, D., Kang, J., Vardoulis, O., Kim, Y., Matsuhisa, N., Oh, J. Y., To, J. W., Mun, J., Katsumata, T., Liu, Y., McGuire, A. F., Krason, M., Molina-Lopez, F., Ham, J., Kraft, U., Lee, Y., Yun, Y., Tok, J. B.-H., and Bao, Z., 2018, “An Integrated Self-Healable Electronic Skin System Fabricated via Dynamic Reconstruction of a Nanostructured Conducting Network,” Nat. Nanotechnol., 13(11), p. 1057-1065.

[13] Chen, X., Dam, M. A., Ono, K., Mal, A., Shen, H., Nutt, S. R., Sheran, K., and Wudl, F., 2002, “A Thermally Re-Mendable Cross-Linked Polymeric Material,” Science., 295(5560), pp. 1698-1702.

[14] Yoshie, N., Watanabe, M., Araki, H., and Ishida, K., 2010, “ThermoResponsive Mending of Polymers Crosslinked by Thermally Reversible Covalent Bond : Polymers from Bisfuranic Terminated Poly ( Ethylene Adipate ) and Tris-Maleimide,” Polym. Degrad. Stab., 95(5), pp. 826-829.

[15] Du, P., Liu, X., Zheng, Z., Wang, X., Joncheray, T., and Zhang, Y., 2013, "Synthesis and Characterization of Linear Self-Healing Polyurethane Based on Thermally Reversible Diels-Alder Reaction,” RSC Adv., 3(35), pp. 1547515482.

[16] Rodriguez, E. D., Luo, X., and Mather, P. T., 2011, "Linear / Network Poly ( $\varepsilon$ -Caprolactone ) Blends Exhibiting Shape Memory Assisted Self-Healing ( SMASH ),” ACS Appl. Mater. Interfaces, 3(2), pp. 152-161.

[17] Liu, J., Liu, J., Wang, S., Huang, J., Wu, S., Tang, Z., Guo, B., and Zhang, L., 2017, “An Advanced Elastomer with an Unprecedented Combination of Excellent Mechanical Properties and High Self-Healing Capability,” J. Mater. Chem. A, 5(48), pp. 25660-25671.

[18] Zeng, C., Seino, H., Ren, J., Hatanaka, K., and Yoshie, N., 2013, “Bio-Based Furan Polymers with Self-Healing Ability,” Macromolecules, 46(5), pp. 17941802.

[19] Hong, G., Zhang, H., Lin, Y., Chen, Y., Xu, Y., Weng, W., and Xia, H., 2013, "Mechanoresponsive Healable Metallosupramolecular Polymers," Macromolecules, 46(21), pp. 8649-8656.

[20] Peterson, A. M., Jensen, R. E., and Palmese, G. R., 2010, "Room-Temperature Healing of a Thermosetting Polymer Using the Diels - Alder Reaction,” ACS 
Appl. Mater. Interfaces, 2(4), pp. 1141-1149.

[21] Chung, C., Roh, Y., Cho, S., and Kim, J., 2004, “Crack Healing in Polymeric Materials via Photochemical [2+2] Cycloaddition,” Chem. Mater., 16(21), pp. 3982-3984.

[22] Amamoto, Y., Kamada, J., Otsuka, H., Takahara, A., and Matyjaszewski, K., 2011, "Repeatable Photoinduced Self-Healing of Covalently Cross-Linked Polymers through Reshuffling of Trithiocarbonate Units,” Angew. Chemie Int. Ed., 50(7), pp. 1660-1663.

[23] Ghosh, B., and Urban, M. W., 2009, "Self-Repairing Oxetane-Substituted Chitosan Polyurethane Networks,” Science., 323(5920), pp. 1458-1460

[24] Burnworth, M., Tang, L., Kumpfer, J. R., Duncan, A. J., Beyer, F. L., Fiore, G. L., Rowan, S. J., and Weder, C., 2011, “Optically Healable Supramolecular Polymers,” Nature, 472(7343), pp. 334-337.

[25] Palleau, E., Reece, S., Desai, S. C., Smith, M. E., and Dickey, M. D., 2013, "Self-Healing Stretchable Wires for Reconfi Gurable Circuit Wiring and 3D Microfluidics,” Adv. Mater., 25(11), pp. 1589-1592.

[26] Xia, N. N., Xiong, X. M., Rong, M. Z., Zhang, M. Q., and Kong, F., 2017, "Self-Healing of Polymer in Acidic Water toward Strength Restoration through the Synergistic Effect of Hydrophilic and Hydrophobic Interactions,” ACS Appl. Mater. Interfaces, 9(42), pp. 37300-37309.

[27] Li, J., Ejima, H., and Yoshie, N., 2016, "Seawater-Assisted Self-Healing of Catechol Polymers via Hydrogen Bonding and Coordination Interactions," ACS Appl. Mater. Interfaces, 8(29), pp. 19047-19053.

[28] Xia, N. N., Rong, M. Z., and Zhang, M. Q., 2016, "Stabilization of CatecholBoronic Ester Bonds for Underwater Self-Healing and Recycling of Lipophilic Bulk Polymer in Wider PH Range,” J. Mater. Chem. A, 4(37), pp. 1412214131.

[29] Adzima, B. B. J., Kloxin, C. J., and Bowman, C. N., 2010, “Externally Triggered Healing of a Thermoreversible Covalent Network via Self-Limited Hysteresis Heating,” Adv. Mater., 22(25), pp. 2784-2787.

[30] Sheridan, R. J., and Bowman, C. N., 2013, "Understanding the Process of Healing of Thermoreversible Covalent Adaptable Networks,” Polym. Chem., 4(18), pp. 4974-4979.

[31] Xu, F., and Zhu, Y., 2012, "Highly Conductive and Stretchable Silver Nanowire Conductors,” Adv. Mater., 24(37), pp. 5117-5122.

[32] Chen, S., Wei, Y., Yuan, X., Lin, Y., and Liu, L., 2016, “A Highly Stretchable Strain Sensor Based on a Graphene/Silver Nanoparticle Synergic Conductive Network and a Sandwich Structure,” J. Mater. Chem. C, 4(19), pp. 4304-4311.

[33] Lipomi, D. J., Vosgueritchian, M., Tee, B. C. K., Hellstrom, S. L., Lee, J. A., Fox, C. H., and Bao, Z., 2011, "Skin-like Pressure and Strain Sensors Based on Transparent Elastic Films of Carbon Nanotubes,” Nat. Nanotechnol., 6(12), pp. 788-792.

[34] Song, P., Qin, H., Gao, H. L., Cong, H. P., and Yu, S. H., 2018, “Self-Healing and Superstretchable Conductors from Hierarchical Nanowire Assemblies," 
Nat. Commun., 9(1), pp. 1-9.

[35] Balazs, A. C., Emrick, T., and Russell, T. P., 2013, "Nanoparticle Polymer Composites : Where Two Small Worlds Meet,” Science., 314(5802), pp. 11071110.

[36] Tee, B. C. K., Wang, C., Allen, R., and Bao, Z., 2012, “An Electrically and Mechanically Self-Healing Composite with Pressure- and Flexion-Sensitive Properties for Electronic Skin Applications,” Nat. Nanotechnol., 7(12), pp. 825-832.

[37] Kubo, B. M., Li, X., Kim, C., Hashimoto, M., Wiley, B. J., Ham, D., and Whitesides, G. M., 2010, "Stretchable Microfluidic Radiofrequency Antennas," Adv. Mater., 22(25), pp. 2749-2752.

[38] Aliev, A. E., Oh, J., Kozlov, M. E., Kuznetsov, A. A., Fang, S., Fonseca, A. F., Ovalle, R., Lima, M. D., Haque, M. H., Gartstein, Y. N., Zhang, M., Zakhidov, A. A., and Baughman, R. H., 2009, "Giant-Stroke, Superelastic Carbon Nanotube Aerogel Muscles,” Science., 323(5921), pp. 1575-1579.

[39] Kim, D., Ghaffari, R., Lu, N., and Rogers, J. A., 2012, "Flexible and Stretchable Electronics for Biointegrated Devices,” Annu. Rev. Biomed. Eng., 14, pp. 113-128.

[40] Zhang, Y., Huang, Y., and Rogers, J. A., 2015, "Mechanics of Stretchable Batteries and Supercapacitors,” Curr. Opin. Solid State Mater. Sci., 19(3), pp. 190-199.

[41] Liu, Y., He, K., Chen, G., Leow, W. R., and Chen, X., 2017, "Nature-Inspired Structural Materials for Flexible Electronic Devices,” Chem. Rev., 117(20), pp. $\underline{12893-12941 .}$

[42] Ma, Y., Feng, X., Rogers, J. A., Huang, Y., and Zhang, Y., 2017, "Design and Application of 'J-Shaped' Stress-Strain Behavior in Stretchable Electronics: A Review,” Lab Chip, 17(10), pp. 1689-1704.

[43] Rogers, J. A., Someya, T., and Huang, Y., 2010, "Materials and Mechanics for Stretchable Electronics,” Science., 327(5973), pp. 1603-1607.

[3944]Kim, C., and Yoshie, N., 2018, "Polymers Healed Autonomously and with the Assistance of Ubiquitous Stimuli: How Can We Combine Mechanical Strength and a Healing Ability in Polymers?,” Polym. J., pp. 1-11.

[4045]Taynton, P., Zhu, C., Loob, S., Shoemaker, R., Pritchard, J., Jin, Y., and Zhang W., 2016, "Re-Healable Polyimine Thermosets: Polymer Composition and Moisture Sensitivity,” Polym. Chem., 7(46), pp. 7052-7056.

[4146]Taynton, P., Ni, H., Zhu, C., Yu, K., Loob, S., Jin, Y., Qi, H. J., and Zhang, W., 2016, "Repairable Woven Carbon Fiber Composites with Full Recyclability Enabled by Malleable Polyimine Networks,” Adv. Mater., 28(15), pp. 2904-2909.

[4247]ASTM, International., 2010, “Standard Test Method for Apparent Shear Strength of Single-Lap-Joint Adhesively Bonded Metal Specimens by Tension Loading (Metal-to-Metal),” ASTM No. D1002 - 10(2019).

[4348] Yu, K., Shi, Q., Li, H., Jabour, J., Yang, H., Dunn, M. L., Wang, T., and Qi, H. J., 2016, “Interfacial Welding of Dynamic Covalent Network Polymers,” J. Mech. 
Phys. Solids, 94, pp. 1-17.

[4449] Yang, H., Yu, K., Mu, X., Wei, Y., Guo, Y., and Qi, H. J., 2016, “Molecular Dynamics Studying on Welding Behavior in Thermosetting Polymers Due to Bond Exchange Reactions,” RSC Adv., 6(27), pp. 22476-22487.

[4550] Yu, K., Qian, S., Wang, T., Dunn, M. L., and Qi, H. J., 2019, “A Computational Model for Surface Welding in Covalent Adaptable Networks Using FiniteElement Analysis,” J. Appl. Mech., 83(9), p. 091002 


\section{Figure captions}

Figure 1. Polyimine synthesis and self-healing process. a) Synthesis of crosslinked polyimine. b) Schematic illustration of the self-healing process of polyimine. Optical images of the polyimine film are shown at the bottom of each frame.

Figure 2. Optical microscopy images of the cross sections of lap-joint polyimine and nanocomposite films. a) Optical microscopy images of the lap-join area of a pure polyimine film before heat press (left), after heat press for 5 minutes (middle) and for 65 minutes (right). b) Optical microscopy images of the lap-join area of a polyimine nanocomposite film with 20\%wt of AgNPs before heat press (left), after heat press for 5 minutes (middle) and for 65 minutes (right). c) Optical microscopy images of the lapjoin area of a polyimine nanocomposite film with 50\%wt of AgNPs before heat press (left), after heat press for 5 minutes (middle) and for 65 minutes (right). The heat press temperature and load were kept constant at $80^{\circ} \mathrm{C}$ and $500 \mathrm{~g}$, respectively. The insets in the middle frames show that the self-healed polyimine strips can hold $1 \mathrm{~g}$ weight after 5 minutes heat press.

Figure 3. Shear strength test specimen design and optical microscopy images of failed interfaces. a) Shear strength test specimen design. Optical microscopy images of the failed interfaces of self-healed pure polyimine (b), 20\%wt AgNP nanocomposite (c) and 50\% AgNP nanocomposite (d) films after heat press at $80^{\circ} \mathrm{C}$ with $500 \mathrm{~g}$ load for 
5 minutes. Optical microscopy images of the failed interfaces of self-healed pure polyimine (e), 20\%wt AgNP nanocomposite (f) and 50\% AgNP nanocomposite (g) films after heat press at $80^{\circ} \mathrm{C}$ with $500 \mathrm{~g}$ load for 65 minutes. At the bottom of each frame shows the optical images of the failed polyimine films.

Figure 4. The dependency of shear strength of self-healed polyimine and nanocomposite films on heat press conditions. a) The shear strength of the self-healed polyimine and nanocomposite films versus heat press time, while the heat press temperature and load were kept constant at $80{ }^{\circ} \mathrm{C}$ and 500 g, respectively. b) The shear strength of the self-healed polyimine and nanocomposite films versus heat press temperature, while the heat press time and load were kept constant at 25 minutes and 500 g, respectively. c) The shear strength of the self-healed polyimine and nanocomposite films versus heat press load, while the heat press time and temperature were kept constant at 25 minutes and $80{ }^{\circ} \mathrm{C}$, respectively.

Figure 5. Characterization of self-healing of conductive polyimine nanocomposites.

a) The lighting circuit of conductive polyimine nanocomposite films. b) An LED light turned on with an original conductive polyimine, went out when the conductive polyimine was cut broken, and turned on again after self-healing of the conductive polyimine film. c) The electrical resistance of the self-healed conductive polyimine nanocomposite films, normalized by the original conductive polyimine nanocomposite films, versus the heat press time, while the heat press temperature and load were kept 
constant at $80{ }^{\circ} \mathrm{C}$ and 500 g, respectively. d) The electrical resistance of the self-healed conductive polyimine nanocomposite films, normalized by the original conductive polyimine nanocomposite films, versus the heat press temperature, while the heat press time and load were kept constant at 25 minutes and 500 g, respectively. e) The electrical resistance of the self-healed conductive polyimine nanocomposite films, normalized by the original conductive polyimine nanocomposite films, versus the heat press load, while the heat press time and temperature were kept constant at 25 minutes and $80{ }^{\circ} \mathrm{C}$, respectively.

\section{Figure sets}

\section{Figure 1}

$\mathbf{a}$

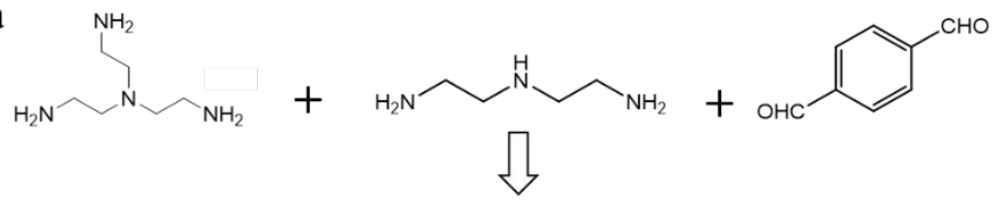

Crosslinked polyimine

b

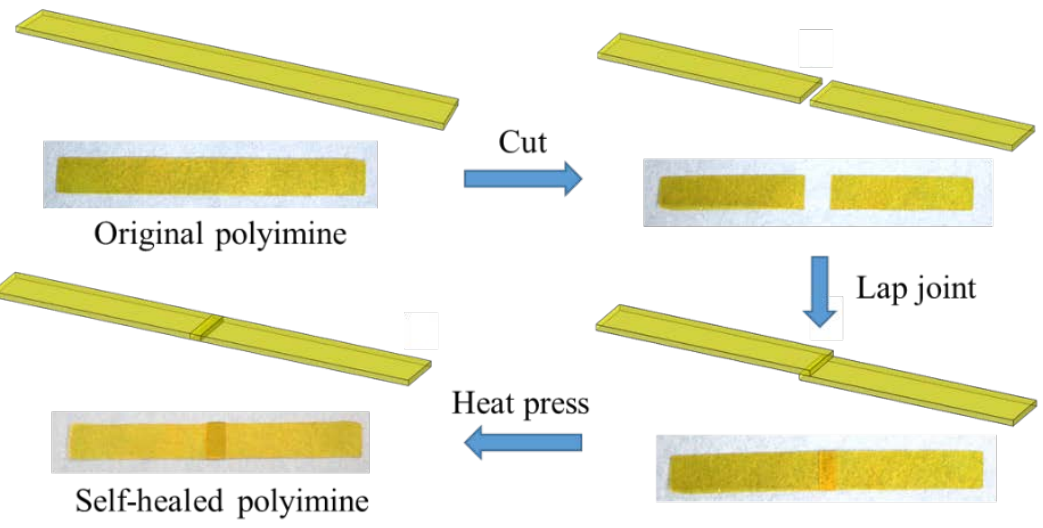


Figure 2

Before heat press

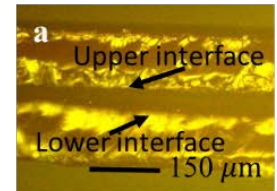
polyimine

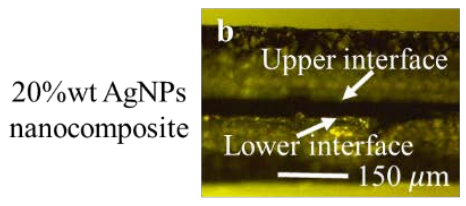

50\%wt AgNPs

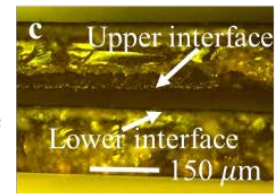

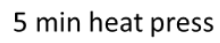
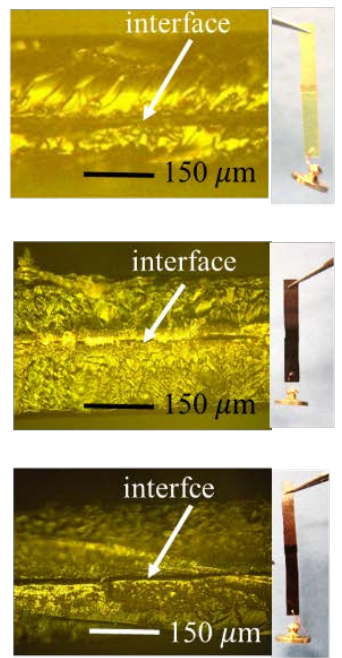

65 min heat press
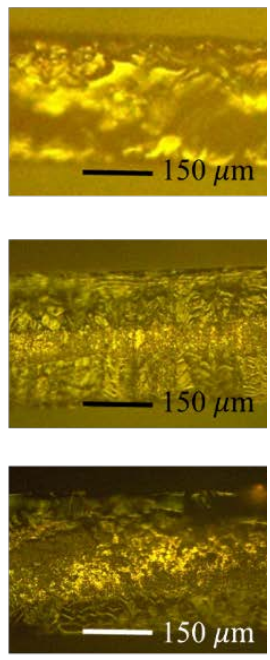

Figure 3

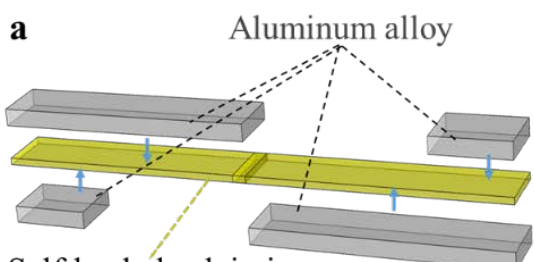

Self-healed polyimine

The pure polyimine
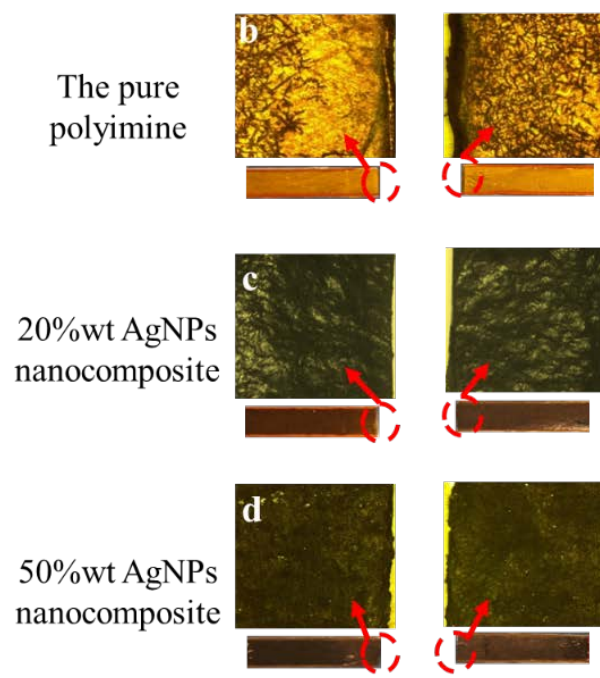

$80^{\circ} \mathrm{C}, 500 \mathrm{~g}$ load, $5 \mathrm{mins}$ heat press
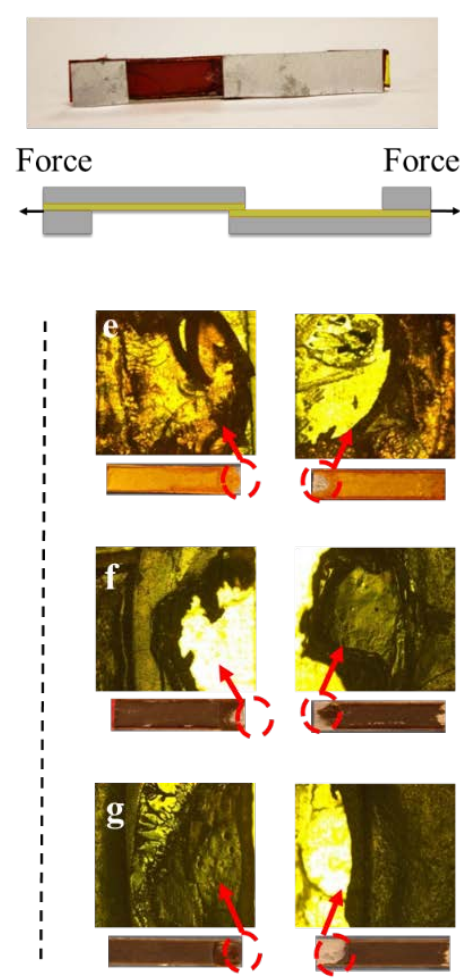

- $500 \mu \mathrm{m} \quad 80^{\circ} \mathrm{C}, 500 \mathrm{~g}$ load, $65 \mathrm{mins}$ heat press 


\section{Figure 4}
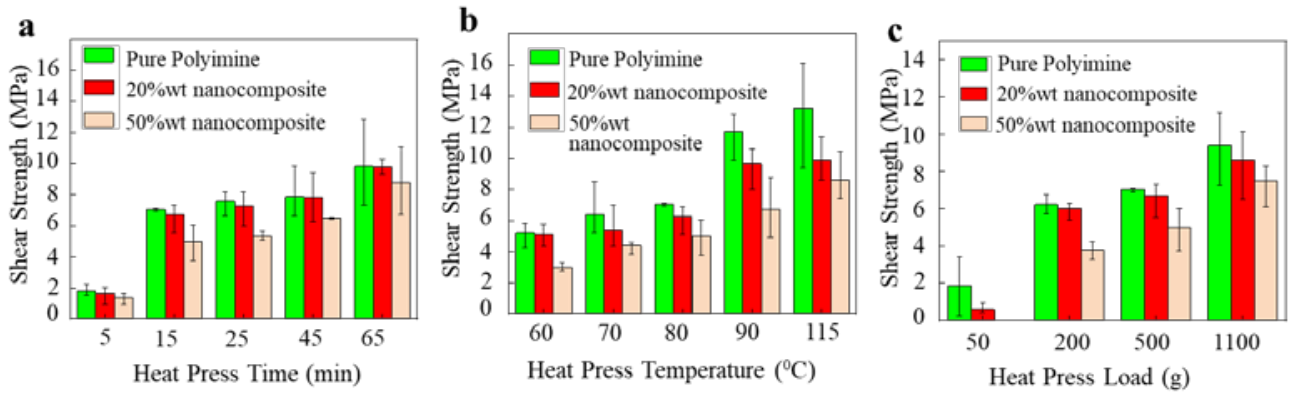

Figure 5
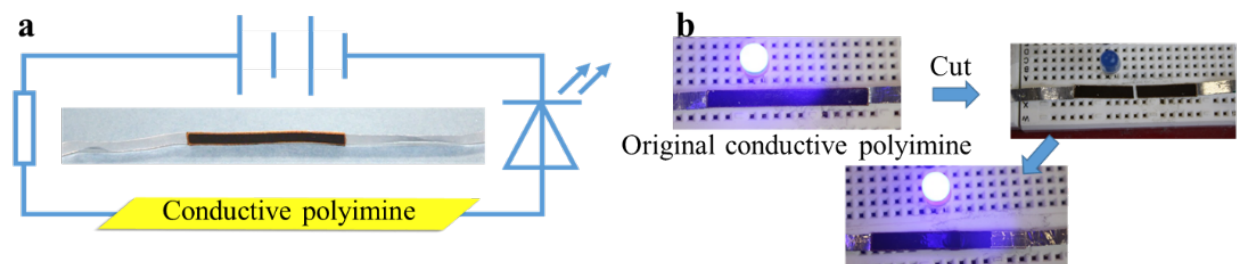

Self-healed conductive polyimine

c

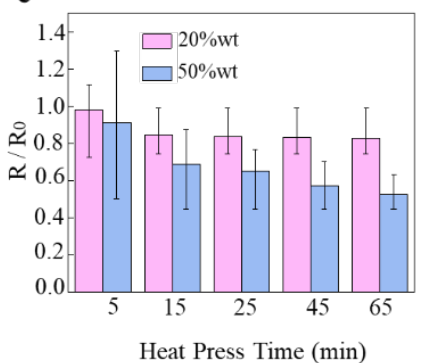

d

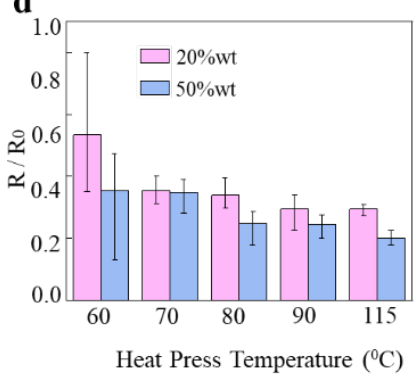

e

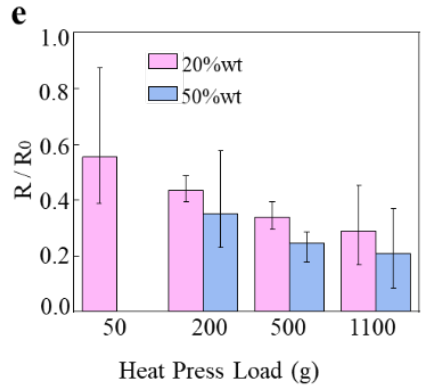

